\title{
Remote medico-legal assessment by telephone during COVID-19: Monitoring safety and quality when documenting evidence of torture for UK asylum applicants
}

\author{
Juliet Cohen' ${ }^{1}$, Bernadette Gregory ${ }^{1}$, Kate Newman' ${ }^{1}$, Emily J Rowe ${ }^{1}$ and \\ Deborah Thackray ${ }^{1}$
}

Key points of interest

- Psychological medico-legal reports can safely be produced by telephone assessment

- The reports can follow Istanbul Protocol Principles

- But they are more likely than face-toface assessments to be incomplete, in terms of both full disclosure of torture experiences and psychological assessment

\section{Abstract}

Introduction: Due to the COVID-19 pandemic, Freedom from Torture developed remote telephone assessments to provide interim medico-legal reports, ensuring people could obtain medical evidence to support their asylum claim.

Method: To audit this new way of working, feedback was collected from the doctors, interpreters, individuals being assessed, and senior medical and legal staff who reviewed the reports. This paper presents findings from the first 20 assessments.

1) Freedom from Torture, United Kingdom Correspondence to jcohen@freedomfromtorture. org
Results: Individuals assessed reported that the doctor developed good rapport, but in 35\% of assessments reported that there were some experiences they felt unable to disclose. In $70 \%$ of assessments, doctors felt that rapport was not as good compared to face-to-face. In the majority of assessments, doctors were unable to gain a full account of the torture or its impact. They reported feeling cautious about pressing for more information on the telephone, mindful of individuals' vulnerability and the difficulty of providing support remotely.

Nevertheless, in $85 \%$ of assessments doctors felt able to assess the consistency of the account of torture with the psychological findings, in accordance with the Istanbul Protocol (United Nations, 2004).

Factors that hindered the assessment included the inability to observe body language, the person's ill health, and confidentiality concerns.

Conclusion: This research indicates that psychological medico-legal reports can safely be produced by telephone assessment, but are more likely to be incomplete in terms of both full disclosure of torture experiences and psychological assessment. The limitations underline the need for a follow-up face-to-face assessment to expand the psychological assessment as well as undertake a physical assessment. 
Keywords: medico-legal, torture, COVID-19, asylum, remote

\section{Introduction}

Freedom from Torture is a human rights charity in the UK providing therapy, support and medico-legal reports for survivors of torture. At the onset of the COVID-19 pandemic in the UK some legal processes for asylum seekers were suspended but others continued. Some substantive asylum interviews and hearings have since restarted. Prior to COVID-19 the Home Office already carried out some interviews by video conference, and it is planned to roll out this option much more extensively.

Rapid change in working practice was required to meet the continuing need for medical evidence for asylum applicants. Guidance was developed for doctors to switch to remote psychological assessments, taking account of the evidence from the rapid change to remote working for the majority of medical appointments at that time, and from more established remote work in mental health services and telephone helplines. While physical examinations had to be suspended, telephone assessments focussed on the account of torture and the psychological assessment but also noted physical symptoms, underlying health conditions and the presence of physical lesions and confirmed the need for face-to-face examination to document physical evidence of torture.

The aim was to ensure people had access to evidence if they needed to progress their asylum claim during lockdown, or make arguments as to why their case could not progress at that time. Reports could also provide other evidence on aspects such as suicide risk, fitness to give evidence or psychological issues which could go to credibility, for example through discussing clinical reasons relating to memory that could account for differences between accounts.
Many asylum seekers either did not have a device with video function or reliable internet access, particularly during the lockdown. There were also initial security concerns about confidentiality with some video platforms. Only Skype for Business was authorised organisationally at this point, due to confidentiality concerns, but this did not support a three-way video function with an interpreter without all users having a business account. The service therefore began with telephone assessments, using three-way calling facilities with interpreters when necessary.

The British Medical Journal has been enthusiastic about remote assessments, declaring that "if the technical connection is high quality, clinicians and patients tend to communicate by video in much the same way as in an in-person consultation" (Greenhalgh \& Kok, 2020: 368). Telephone consultations have been used in General Practice for some time. However, others have sounded strong notes of caution for such a generic endorsement of the use of technology. Royal College of Psychiatrists guidance states that "For initial consultations (where the patient and clinician are unknown to each other), remote consultations may be even more challenging. Despite this, the alternative of no consultation at all is not preferable and we recommend that initial remote consultations go ahead where possible.... It remains the case that these consultations are limited and those with lack of digital literacy or no access to digital platforms must not be disadvantaged, nor should those who are unconfident about using the technology" (Royal College of Psychiatrists, 2020).

There is a key difference between consultations taking place in the National Health Service, to address current clinical need, and a medico-legal report (MLR) examination, where the person's past experiences of torture, and other experiences of trauma, must be explored in order to correlate the level of con- 
sistency with current clinical findings. MLR assessments cause a degree of anxiety for the person in any situation, knowing they will be asked to recount the experiences they may be desperate to forget. In light of this, caution was needed in balancing any risks to individuals who, during lockdown, would be more likely to be isolated and unable to access support networks, against the need for an assessment when the doctor could less readily assess levels of distress or re-traumatisation compared to a face-to-face assessment. After MLR examinations, some people feel an increase in distress and experience a higher frequency and intensity of PTSD symptoms. In normal times they can visit their family doctor or counsellor, see friends, or gain comfort from attending their place of worship. Few if any of these options were available to them during the lockdown. The MLR process may need to go into exactly those areas cautioned against by the Royal College of Psychiatrists and the guidance given to therapists. Therefore, it was paramount not only to have guidance in place for doctors but also to carry out an audit so that the safety and quality of the assessments could be assessed almost immediately.

The objective of this study is therefore to assess the extent to which telephone assessments can evaluate evidence of torture while monitoring individuals for risk of harm in the assessment process.

\section{Method}

1. Guideline development: Detailed guidance was developed for doctors undertaking remote assessments, including when a remote assessment might be unsuitable.

2. Ethics: Freedom from Torture research ethics committee approved the project. Throughout the process the precept 'first do no harm' was kept in mind. This guided a cautious approach regarding the risk of re-traumatisation and exacerbation of mental health conditions to those examined remotely, and our assessment of the quality of evidence produced in this way.

3. Training: Doctors were provided with the new guidance and a short remote training session was held. All the doctors had more than five years post-qualification experience and most had many years broad general medical experience. In addition, all had previously undertaken a comprehensive course of specific training in assessment and documentation of torture provided in-house, and attend regular update training.

4. Risk assessment: For each referral, factors were considered that might make a remote assessment unsuitable for that person. These included:

- Hearing difficulty

- Learning difficulty or disability

- Other significant cognitive disability

- Severe psychiatric disorder or being under the care of a mental health team, for example if the person was known to dissociate, had a diagnosis of psychosis or a high risk of suicide

- Single parent with dependent children, or children present in their accommodation

- Age under 18

- Any other factor identified from the referral documents

Additional information was gathered from the person's therapist, if with Freedom from Torture, or legal representative, to inform this risk assessment.

5. Choice: Individuals considered suitable, and their legal representatives, were offered the choice of a remote assessment to produce an interim MLR, followed by a 
face-to face appointment to complete the report when conditions allow, or a place on the waiting list to have a full report once face-to-face assessment was possible.

6. Sample: All assessments were carried out between April and July 2020 with individuals in their accommodation, during the complete lockdown period. Assessments were undertaken by a pool of doctors, interpreters and lawyers, with some working with a single client and some with several clients. The age range of clients was 23-53 years and 19 out of 20 were male. Clients came from the following countries (table 1):

7. Preparation: Each case file was prepared by an MLR legal officer, summarising key issues to be addressed. The doctor read the documents themselves and a discus-

\begin{tabular}{ll}
\hline Table 1. Countries of origin & \\
\hline Afghanistan & 2 \\
Angola & 1 \\
Cameroon & 1 \\
DRC & 2 \\
Egypt & 1 \\
Ethiopia & 1 \\
Kenya & 1 \\
India & 1 \\
Iraq & 1 \\
Pakistan & 2 \\
Sri Lanka & 4 \\
Sudan & 2 \\
Zimbabwe & 1
\end{tabular}

sion took place prior to the assessment.

8. Process: Each assessment was carried out over two phone calls, each up to two hours in length. The doctor first assessed the person's health and vulnerability in the pandemic and made a risk assessment. If safe to do so, they continued the assessment to take an account of the torture and its impact on the person's health, and produced a draft report.

9. Review: Each report was reviewed by a senior doctor and lawyer before the doctor completed the report.

10. Audit: Feedback was collected from the doctors, interpreters, the individuals assessed and the medical and legal staff who reviewed the reports. For comparison, feedback was also sought from individuals who had had their MLR produced prior to the COVID-19 lockdown, either with all face-to-face appointments or in a few cases, some face-to-face appointments with a phone call to complete the assessment.

11. Analysis: A simple analysis of the data is made. Although results are quoted as percentages, it is acknowledged that numbers are relatively small and results comprise both quantitative and qualitative information. Presentation in this format however aims to provide clarity in the comparisons being made.

12. Future follow-up: Follow up face-to-face appointments will be offered, when this is possible, for the physical examination and for any areas that could not be covered in the remote assessment, or where an update is needed.

\section{The Guidance}

The guidance for remote assessments comprised details on the administrative process for setting up three-way calls with an interpreter, the safety-net process for first assessing the person's vulnerability and current health before proceeding to take the torture history, and advice on how to adapt the in-person assessment and examination for the remote 
process. The guidance considered both telephone and video calls, but as discussed in the introduction, in this data set only telephone assessments are reported.

The principle of minimising risk guides doctors to proceed with caution, checking the person understands the purpose of the call, gives their consent, understands who will call back if the call drops out, and that they are in a suitably private location to undertake the assessment. The doctor obtains key contact information in case of emergency. A discussion is held about what to do if the person feels upset and overwhelmed or needs a break.

The doctor checks the person's understanding of COVID-19 symptoms and what to do if they become unwell. This is followed by a general review of the person's current health and a risk and safeguarding assessment, checking they feel safe and are able to look after themselves and their dependants. Flexibility is advised once the assessment proceeds to assess evidence of torture, for example by considering starting with the current psychological examination before working backwards to an account of the torture, depending on the doctor's assessment of how the person is responding. Doctors were advised to use some techniques more often and more overtly than when face-to-face, such as checking with the person how they are feeling, asking about silences, demonstrating active listening by summarising or repeating back part of what the person has said, signposting the next topic to be discussed, and using more direct questions to keep the person focussed.

\section{Feedback surveys}

After each assessment, the doctor and interpreter completed a survey. Once the report was completed, the medical and legal reviewers also recorded their feedback. The feedback included an evaluation of the extent to which the doctor felt able to gather details of the torture and current psychological state, the extent to which they were able to assess the consistency of the account of torture with the clinical findings in accordance with the Istanbul Protocol. It also included other aspects relevant to their asylum claim such as suicide risk assessment, identifying treatment needs, and fitness to give evidence. A different doctor called the person assessed a few days later to obtain his or her feedback.

The surveys comprised a mix of open and closed questions and responders were invited to add free text to elaborate on their answers. The survey questions asked are in appendix 1 .

Table 2. Doctors' feedback

\begin{tabular}{llll}
\hline & $\begin{array}{l}\text { Fully; as if } \\
\text { face to face }\end{array}$ & Partially & $\begin{array}{l}\text { Not at } \\
\text { all }\end{array}$ \\
\hline $\begin{array}{l}\text { To what extent were you able to obtain an account } \\
\text { of torture? }(2 / 20 \text { excluded as torture account already } \\
\text { taken) }\end{array}$ & $5 \%$ & $67 \%$ & $28 \%$ \\
$\begin{array}{l}\text { To what extent were you able to assess the impact of } \\
\text { torture? }\end{array}$ & $20 \%$ & $75 \%$ & $5 \%$ \\
$\begin{array}{l}\text { To what extent were you able to make an assessment } \\
\text { of the current psychological condition? }\end{array}$ & $75 \%$ & $25 \%$ & \\
$\begin{array}{l}\text { To what extent were you able to consider consistency } \\
\text { r }\end{array}$ & $60 \%$ & $25 \%$ & $15 \%$
\end{tabular}




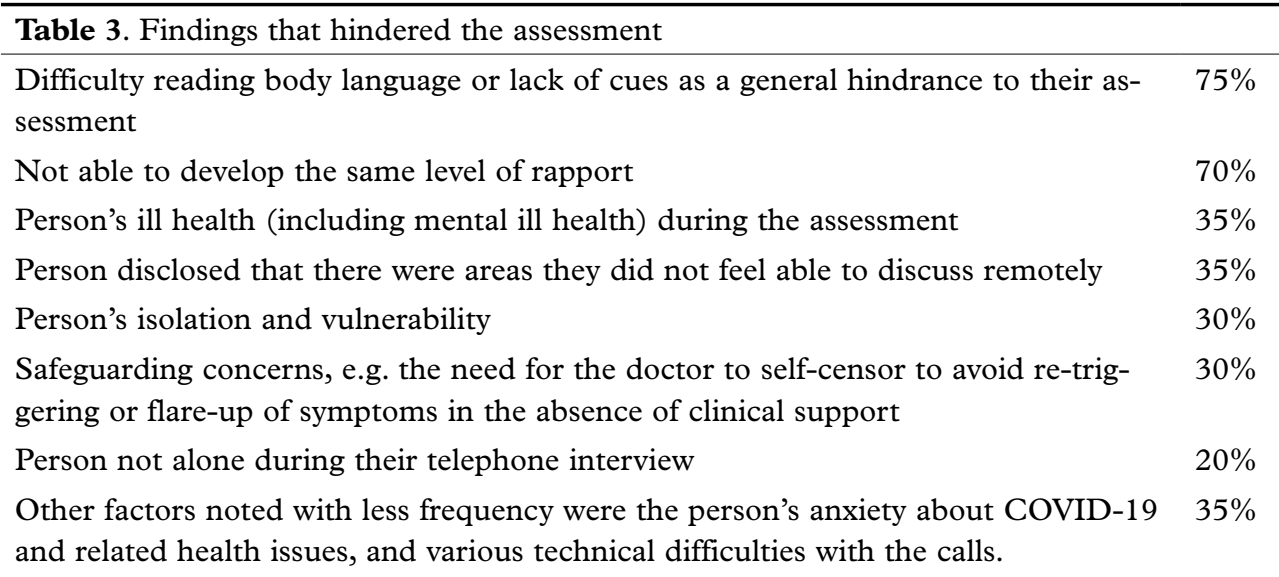

\section{Results}

Doctors' feedback is shown in table 2. Table 3 summarises findings that were felt to hinder the assessment.

Factors noted to have helped the assessment

- A good legal briefing prior to the first appointment allowed doctors to maximise the value of the time spent on the calls and prioritise areas to focus on.

- A good interpreter.

- Two phone calls helped develop rapport and allowed flexibility in responding to the individual's presentation, for example the first call was often shorter, and more detail was gathered in a second longer call.

- Ability to make a brief third call to clarify a specific issue.

- Flexibility in offering breaks.

- Flexibility in approach, for example approaching the torture history later in the second session when the person's psychological state and risk of re-traumatisation had been assessed and better rapport established.
Feedback from people examined face-to-face: Seven responses from people who had had a face-to-face appointment before COVID-19 were gathered for comparison with those of people examined remotely. Comments included that

- panic attacks were exacerbated by the whole MLR process and nightmares and distressing memories increased.

- One individual who had two face-to-face appointments and one remote, found it hard to listen and concentrate on the phone call.

Comments on rapport included that

- "the doctor was kind and listened to me",

- "I could talk openly, valued being heard",

- "the office space and the environment created by the doctor made me feel comfortable which made the assessment easier"

- "I felt I could say everything I wanted to".

One person commented "Some questions were hard to answer, didn't want to answer, felt I had to answer".

\section{Feedback from people examined remotely:}

20 feedback responses were received from those examined by telephone (table 4 ). 
Table 4. Preference for face-to-face vs telephone assessments

$\begin{aligned} & \text { Better to have a face-to-face as- } \\ & \text { sessment }\end{aligned}$
$\begin{aligned} & \text { Face-to-face or telephone the } \\ & \text { same }\end{aligned}$
$\begin{aligned} & \text { Better to have telephone assess- } \\ & \text { ment }\end{aligned}$

Comments that were in common with those made about face-to-face assessments

- "Went OK", "felt comfortable"

- "Talking about the past creates terrible feelings", "more depressed"

- 'Able to say everything I wanted to'

- There were many compliments to the doctors, that they were comforting, understanding, offered support, helpful

\section{Other comments}

- Five individuals felt it would have helped if they could show the doctor their body.

- One individual felt that a doctor could have understood better how they were struggling mentally and emotionally if they could have seen them.

- Four said they feared anxiety, breaking down or panicking if the assessment had been face-to-face.

- One individual found it difficult to talk on the phone for a long time, difficult to maintain concentration.

- One person had to find a private place in a park to be able to talk.

\section{Comments on disclosure}

- $80 \%$ of individuals indicated that they had said all they wanted to.

- $40 \%$ of individuals remarked that it was difficult talking about past experiences.
- "There are a lot of things I chose not to talk about".

- "I said everything I wanted to say; there are some things I didn't share with the doctor because I didn't want to share them with anyone'.

- "I was able to say everything that I wanted to. I admit that I was, however, hesitant to tell everything. I kept a few things back. I was not $100 \%$ open, but more than I would have been face-to-face".

- "I think face to face would have been more difficult - telling all my experiences. I am not good with face-to-face interviews - I get anxious. Through the phone interview I was able to say everything".

- "After 2 days of phone interviews I felt emotionally relieved - it helped me a lot".

\section{Interpreters feedback}

\begin{tabular}{ll}
\hline Table 5. Interpreters' responses & \\
\hline Communication and rapport were & $42 \%$ \\
not negatively affected by the & \\
remote process \\
Communication and rapport were $\quad 47 \%$ \\
somewhat negatively affected by the \\
remote process \\
Communication and rapport were \\
helped by the remote process
\end{tabular}

19 responses received from interpreters who interpreted during the telephone assessments are shown in table 5 .

Comments included:

- 8 respondents noted that video call would be better; among the reasons cited were the loss of body language.

- 2 respondents noted difficulties maintaining a good connection. 
- 1 respondent valued the pre-appointment briefing and post-meeting review with the doctor, without the individual.

- 1 respondent noted there was a point where it sounded like the person was sobbing, but then composed himself, whereas face-toface it would have been easier to know what was happening.

\section{Reviewer feedback}

The reviewer feedback surveys were predominantly in a free text format. The percentages quoted below should therefore be viewed as a broad indication of the findings.

There were responses by legal reviewers for 14 cases. In $35 \%$ of the responses, the legal reviewer noted within their comments that the doctors' fears around re-traumatisation or significant distress of the person limited the assessment. No other specific limitations from a legal perspective were noted.

In 15 responses by medical reviewers, the free text comments gave a clear indication of the linkage between the psychological findings with the torture described (see Table 6).

Reviewers also found that doctors were able to make an assessment of the possibility of fabrication of the psychological findings.

\section{General issues}

Confidentiality: There were two cases, where despite best efforts, the person had young children present which limited the extent to which the doctor could explore the account of torture and its impact. In one case the person was in a shared room and their roommate could be heard in the background, but the person wished to continue as they thought their roommate was asleep. In another case the person was taking the phone call out of doors, so again not in good conditions of privacy.

Unsuitability for remote process: In a number of cases the person was not deemed suitable for a remote assessment and was placed on a waiting list for a full face-to-face report when this becomes possible. The numbers excluded were relatively high initially, as a proportion of total referrals, but this proportion reduced as legal representatives understood the COVID situation and the remote assessment process better. The total number of referrals deferred for a face to face assessment during the period of this study was 10 , The reasons for this were (in some cases more than one reason applied):

- In 7 cases the person's mental health made them too vulnerable for a telephone assessment

- In 2 cases the documentation of the person's scars was the most important element, so they wanted to wait until a full face-to-face examination could be done.

- In one case young age was the excluding factor.

\section{Discussion}

The results indicate that psychological medico-legal reports can be carried out by telephone assessment, but their scope may be limited by this method of communication.

\section{Disclosures}

The range of feedback from individuals sug-

Table 6. Linkage of psychological findings

\begin{tabular}{lccl}
\hline & Good & Partial & $\begin{array}{l}\text { Very } \\
\text { limited }\end{array}$ \\
\hline Linkage of psychological findings with the torture described. & $53 \%$ & $24 \%$ & $23 \%$
\end{tabular}


gests some people found telephone assessment easier for disclosure, and others found it perhaps easier to withhold information they did not wish to share. Both those assessed remotely and face-to-face may choose not to disclose everything they can.

In many cases the doctor said that they were not able to gain a full history of the torture or its impact during the assessment. Doctors reported feeling more cautious about pressing for distressing information while on the telephone, mindful of individuals' vulnerability and the difficulty of providing support remotely. Complete disclosure may not be needed if there is already a detailed account elsewhere or if the doctor felt they had enough information to complete the psychological assessment. Reviewing the torture history will need to be an integral part of the later faceto-face assessment as more detail may be be needed to make an assessment of the physical findings. Additional disclosures may be made during subsequent face-to-face assessments and monitoring changes in disclosure patterns will form part of the ongoing audit.

\section{Safeguarding and rapport}

Individuals' feedback indicated that they felt doctors were successful in safeguarding their well-being as far as possible and developing rapport, such that they felt listened to, understood and that the doctor was caring and helpful. This matched the feedback from individuals who had face-to-face assessments. Both groups reported that re-experiencing symptoms such as flashbacks and intrusive memories of torture increased in the period after an appointment.

There were differences in the perception of rapport. The majority of those assessed reported that they could say everything they wanted to. In contrast, doctors often felt that rapport was not as good as when they examine people face-to-face. Interpreters also felt that rapport was not as good and took longer to establish. For most of those being assessed, this was their first experience of an MLR assessment, whereas the doctors and interpreters have prior experience of face-to-face assessments. Many doctors commented that the absence of visual cues made it more difficult to assess the level of rapport and so they felt more cautious in their questioning. Unfamiliarity and anxiety with new technology for three-way calls may also have played a part as technical issues were problematic in some of the first cases. With learning and experience and the smooth running of the technical processes, doctors may find their perception of rapport increases.

Several doctors commented that rapport improved in the second telephone call and that they waited until rapport was better established before approaching the torture history. It seems likely therefore that for some at least, there would have been less disclosure in an assessment format with just a single telephone call.

\section{Confidentiality}

The presence of others near the person in some assessments, including (in two cases) young children, illustrates the difficulties of ensuring confidentiality for remote assessment. This may also be a further factor limiting disclosure in some cases.

\section{Assessing the consistency of the psychological symptoms with the torture allegations}

Assessing the consistency of the psychological symptoms with the torture that the person describes is a key component of a psychological MLR as set out in the Istanbul Protocol paragraph 287. In spite of the limitations doctors faced in obtaining a full history of torture and its impact, in $60 \%$ of assessments the doctor 
reported that they were able to fully assess the consistency of the psychological symptoms with the account of torture given, and a further $25 \%$ were able to make a partial assessment. These findings were mirrored in the feedback of medical reviewers.

This disconnect can be explained by a number of reasons. In many cases doctors had established enough symptomatic detail to demonstrate how they reached diagnoses of Post-Traumatic Stress Disorder (PTSD) and/or depression and to demonstrate how the psychological findings linked to the torture account, for example through the content of nightmares and flashbacks and the timeline of onset and exacerbations of symptoms. Doctors routinely discussed other possible causes of the psychological symptoms as part of this assessment. In some cases, additional history may have been available, for example from a Home Office interview or witness statement, to feed into the assessment. The inability to disclose was often highly relevant to the psychological findings. For example, avoidance behaviour in PTSD or shame resulting from sexual torture provided strong evidence of the psychological impact of torture events even when a full history could not be obtained.

\section{Scope of remote assessments}

Review of the reports showed that doctors were able to make detailed self-harm and suicide risk assessments, which included the current risk and consideration of suicide risk if the person is returned to their country of origin. Legal representatives were sometimes seeking specific information in these areas.

If there were differences between accounts, the doctor discussed any clinical reasons relating to memory that could account for these and any inappropriate clinical judgments

made by decision-makers. In all cases doctors considered whether there was any clinical evidence to suggest fabrication of the psychological symptoms. These considerations provide key evidence to inform the assessment of credibility by the decision makers.

Information in the reports may also be useful for the interviewing officer or tribunal in planning how to conduct an interview or hearing with a vulnerable person, on video link or in person. Doctors routinely considered the fitness of the person to be interviewed or give evidence remotely, and in some cases the doctor was able to make specific recommendations to help with this.

\section{Assessing the remote process}

It was especially valuable to learn from individuals going through the assessment. When changing a process, the duty of care for, and potential harm to, vulnerable individuals has to be kept in mind. Harm may also be caused if the quality of evidence gathered is affected. Our results to date indicate that $85 \%$ of doctors felt able to make an assessment, to a greater or lesser extent, of the consistency of their psychological findings with the torture account given. However, there was a consensus that accounts were likely to be partial, and findings incomplete, notwithstanding the inevitable absence of the physical findings, which had to be pended until a face-to-face examination is possible.

In other areas of medicine there has been relatively little concern expressed about the quality of remote consultations. In the UK both hospital and family doctor consultations have been going ahead remotely, with many commenting they will continue this in the future due to the time saved and increased access. Mental health work has been done remotely in some services for some time, and the use of crisis phone lines is well-established.

In the UK, courts have increasingly moved to remote hearings over several years for bail 
hearings, sentencing hearings, and other work, citing the time and money saved as a key driver. The Home Office is now rolling out remote asylum interviews after a pilot, and it appears that even as COVID-19 related restrictions ease and the threat from the pandemic decreases, this will increase. However, research shows that an adverse credibility assessment is more likely when giving remote evidence (Bail Observation Project 2013), and that people are less likely to get bail, and more likely to get longer sentences (Transform Justice, 2017). This is the case particularly if they have a hearing difficulty, cognitive or mental health problems, or need an interpreter (Transform Justice, 2017). It is evident that more than one of these factors will particularly affect survivors of torture seeking asylum in the UK, as almost all need an interpreter and the prevalence of mental health conditions is high. Indeed, hearing difficulty and significant cognitive or other mental health problems were among the exclusion criteria considered in this study. A study comparing telephone and face-to-face advice in social welfare and legal aid (Burton 2018) found that while the telephone offers convenience and accessibility, those with more complex and serious needs as well as those where trust and rapport are more important are much better served by face-toface communication.

In the examinations reported in our study, the doctors had the advantage of careful case preparation with a legal officer. In contrast to non-medical staff in Home Office or tribunal settings, the doctors have specific clinical skills and experience. This enables them to alter a line of questioning when appropriate to reduce a person's distress, to gauge at what point to approach talking directly about traumatic experiences, to use breaks and/or stop appointments early when a person becomes distressed, and to use second appointments for further development of rapport. In the event that flashbacks or other dissociative episodes are triggered, where the person loses touch with the here and now, they have expertise in using grounding techniques (clinical techniques to bring the person back into the present).

Undergoing asylum interviews or giving evidence to a Court by video link cannot be directly compared with telephone assessments. Medico-legal report examinations, while including a consideration of fabrication of the clinical findings, are not credibility assessments. However, some factors will be common to these different situations, such as the potential for the level of rapport to impact on disclosure, and the importance of making special considerations for those likely to be disadvantaged by the remote nature of the assessment due to cognitive impairment or mental health condition.

In view of these concerns, Freedom from Torture together with the Helen Bamber Foundation (a charity supporting refugees and asylum seekers who have experienced extreme human cruelty, such as torture and human trafficking) have drafted recommendations to the Immigration Tribunals drawing attention to the added vulnerability of victims of torture and other asylum seekers if they are interviewed or required to give evidence remotely. These include the note that "expert evidence that is produced, including medico-legal reports, may be interim only. Evidence of torture survivors' physical injuries may be inaccessible but that in the current circumstances benefit of the doubt should be applied and interim evidence may be sufficient to substantiate a claim" (Freedom from Torture and Helen Bamber, 2020:4).

\section{Limitations of this study}

Numbers: This study reports on the first 20 remote interim medico-legal reports from 
telephone assessments., Findings are limited to this relatively small number and to this medium, limiting the extent of analysis of the data.

Completing the reports: A follow-up paper is indicated, evaluating how the reports change after a face-to-face assessment when the further clinical findings can be considered together with those from the remote assessment. It will be particularly interesting to compare those cases where disclosure was limited with the findings from the face-to-face assessment to see if further disclosures are subsequently made and to examine what impact this has on the report conclusions.

Physical assessment: Once this is added to the reports, it will be interesting if, and to what extent, the report's conclusions may alter.

Outcomes: At this stage, the outcomes in terms of asylum decisions made on the basis of this evidence are not known, or how these psychological reports will be considered. A further paper evaluating this is indicated, once these decisions are made, but may be long-delayed depending on how quickly asylum decisions are processed and appeals heard and to what extent the interim medico-legal reports are relied upon in determining the claims.

Continuing assessments during the era of COVID 19: Although yet to be fully evaluated, early experience with video assessments confirms that not all asylum seekers have access to suitable devices or have the digital literacy or reliable internet access to enable this type of remote assessment. A further audit will aim to compare the telephone assessments with video assessments, now that these have become feasible for some.

\section{Conclusion}

Vulnerable survivors of torture are even more vulnerable if they are not able to access the medical evidence needed to support their asylum application. In meeting this challenge, Freedom from Torture has sought to mitigate their vulnerability by providing a remote health assessment and an interim psychological-only medico-legal report, while minimising distress and the risk of re-traumatisation.

The results of our audit so far show that psychological medico-legal reports can safely be produced by telephone assessment, but are more likely than face-to-face assessments to be incomplete in terms of both full disclosure of torture experiences and psychological assessment. In many cases high quality evidence can be produced that follows the Istanbul Protocol Principles and addresses other key clinical issues in the claim. In cases where significant limitations have been identified, the reports should be regarded as interim.

Providing these reports has ensured that survivors of torture have access to vital medical evidence for their asylum application during the COVID-19 restrictions. Freedom from Torture continues to learn and strive for the highest quality evidence through different types of remote assessment but considers telephone assessment remains crucial to avoid the 'digital discrimination' highlighted by the Royal College of Psychiatrists.

\section{References}

Bail Observation Project. (2013). Still a travesty: Justice in immigration bail hearings. https:// bailobs.files.wordpress.com/2015/03/2nd-bopreport.pdf

Burton, M. (2018). Justice on the line? A comparison of telephone and face-to-face advice in social welfare legal aid. Fournal of Social Welfare and Family Law, 40 (2). pp. 195-215. https://eprints.mdx.ac.uk/23831/1/ Justice \%2520on\%2520the \%2520Line \%2520$\% 2520$ author\%2527s\%2520version.pdf

Freedom from Torture and Helen Bamber. (2020). Tribunal courts and COVID recommendations. hhttps://www.freedomfromtorture.org/sites/ default/files/2020-08/Tribunals-courts-andCOVID-recommendations-Final.pdf

Greenhalgh, G. \& Kok, G. (2020). COVID-19: 
a remote assessment in primary care. British

Medical fournal 368:m1182. https://doi. org/10.1136/bmj.m1182

Royal College of Psychiatrists. Digital COVID 19 guidance for clinicians. 21.05.20 https://www. rcpsych.ac.uk/about-us/responding-to-covid-19/ responding-to-covid-19-guidance-for-clinicians/ digital-covid-19-guidance-for-clinicians

Seuren, L. M., Wherton, J., Greenhalgh, T., Cameron D., A'Court, C. \& Shaw, S. E. (2020) Physical examinations via video for patients with heart failure: qualitative study using conversation analysis. F Med Internet Res 22:e16694

https://www.jmir.org/2020/2/e16694/

Transform Justice. (2017). Defendants on videoconveyor belt justice or a revolution in access. http://www.transformjustice.org.uk/wp-content/ uploads/2017/10/Disconnected-Thumbnail-2.pdf

United Nation (2004) Istanbul Protocol Manual on the effective investigation and documentation of torture and other cruel, inhuman or degrading treatment or punishment.

\section{Appendix 1}

\section{Feedback questions}

\section{MLRS Remote Assessments Doctors Survey} Email:

1. Your name (first name and surname):

2. MFID number:

3. Date of last remote appointment:

4. How many appointments did you have?

5. Were your calls made with video or voice only?

If you answered a mix in the above question, please state the reasons why and what proportion of the calls were voice and video:

6. Was the individual alone, or accompanied? Please provide further details if relevant:

7. To what extent were you able to obtain an account of torture?

Please provide further details if you are able to:

8. To what extent were you able to assess the impact of torture?

Please provide further details if you are able to:
9. To what extent were you able to make a psychological assessment of the current condition?

Please provide further details if you are able to:

10. To what extent were you able to consider consistency of the current psychological condition with the account of torture given?

11. If your answer to the above question was that you were not able or were only partly able to consider consistency of the current psychological condition with the account of torture given, do you believe this is because you were not able to meet with the individual in person?

Please provide further details if you are able to:

12. To what extent were you able to address other specific instructions from the legal representative, such as unfitness to give evidence?

Please provide further details if you are able to:

13. If your answer to the above question was that you were not able to address some or any of the legal representative's instructions, was this a result of not being able to meet with the individual in person?

Please provide further details if you are able to:

14. What factors helped or worked well in the assessment? For example, having a prebriefing, taking breaks, a second/follow up call, or perhaps you have specific tips for others to use?

15. What factors, if any, hindered the assessment?

16. Did the person identify any areas they did not feel ok to disclose or discuss remotely?

Please provide further details if you are able to:

17. How much contact time with the person was spent on issues not directly related to assessing evidence of torture (and not including consent and other preliminaries) - e.g. identifying current non-torture-related healthcare needs, providing 
support and information?

Please provide further details if you are able to:

18. How much contact time with the person was spent on issues not directly related to assessing evidence of torture, including identifying current non-torture-related healthcare needs, providing support and information, that were _directly related to the COVID-19 pandemic_?

Please provide further details if you are able to:

19. How effective did the interventions mentioned in the two questions above appear to be? (Skip this question if not applicable) Please provide further details if possible, or state if it was not clear how effective the interventions were.

20. Did these interventions affect the process of the report examination?

Please provide further details if possible:

21. Did you feel that you were able to build rapport with the person and establish a trusting professional relationship with the person to the same extent as if you met in person?

Please provide details if possible, including any positive factors that enabled you to build a rapport, such as having a second appointment booked or expectation of a possible physical meeting in future, etc:

22. If you have anything else you would like to add, including any feedback on using this survey, please do so here:

\section{MLRS Remote Assessments Individual Feedback Survey}

Email address:

1. Your (doctor's) name (first name and surname):

2. MFID number:

3. Name of the doctor who carried out the remote assessment:

4. Date of last remote appointment (if known):

5. Did the individual give their verbal consent to record and use their answers here for research, policy work, media / communications work, teaching \& training, and fundraising (please click 'other' and provide details if only partial consent was given)?

6. How did you feel the assessment went? Is there any specific feedback you would like to give us?

7. Was there anything that particularly helped you communicate via video link/ phone? (e.g. something the doctor said?)

8. Was there anything in particular that made it difficult?

9. Were you able to say everything that you wanted to? If not, can you say why?

10. If you had been able to have a face to face appointment instead, in what way do you think that might have been different?

11. Anything else you would like to say?

\section{MLRS Face-to-Face Individual Feedback} Survey

Email address:

1. Your name (first name and surname):

2. MFID number:

3. Name of the doctor who carried out the assessment:

4. Date of last appointment (if known):

5. Did the individual give their verbal consent to record and use their answers here for research, policy work, media / communications work, teaching \& training, and fundraising (please click 'other' and provide details if only partial consent was given)?

6. How did you feel the assessment went? Is there any specific feedback you would like to give us?

7. Was there anything that particularly 
helped you to communicate at your appointments? (e.g. something the doctor said?)

8. Was there anything in particular that made it difficult?

9. Were you able to say everything that you wanted to? If not, can you say why?

10. If you had to have your appointment by video or by phone, in what way do you think that might have been different? [For part-face-to-face, part-remote individuals please indicate this was the case, and ask instead: "If you had to have your appointments only by video or by phone, or if you had been able to have face to face appointments, in what way to you think that might have been different?"]

11. Anything else you would like to say?

\section{MLRS Remote Assessments Interpreters Survey}

1. Your name (first name and second name):

2. Name of doctor you worked with:

3. Date of the last interpreting session with this MLR doctor (if known):

4. To what extent did you feel communication and rapport building were affected by the remote process?

5. Please provide further details if you can:

6. What could be done to improve the process of remote interviewing?

7. What challenges, if any, did you yourself face in providing interpreting services for the MLR service remotely?

8. Any other comments, concerns or suggestions?

\section{MLRS Remote Assessments Medical Reviewers Survey}

Email address:

1. Reviewer's name:
2. MFID number:

3. Date of last remote appointment (if known):

4. Was there a particular area of concern in the report on which you needed to comment?

5. Was the reviewing support required different to that of a face-to-face MLR - for example dealing with the limitations of a remote assessment?

6. To what extent has it been possible to link the person's psychological findings to torture?

7. To what extent has the doctor been able to address fabrication?

8. Apart from the physical examination, what points will need following up in a face to face assessment (please ensure you have included this in your review)?

9. Please outline any areas of best practice you picked up? (Please copy any good examples of wording into the 'good examples of wording' document after checking consent and appropriate redaction).

10. Anything else you would like to say?

MLRS Remote Assessments Legal Reviewers Survey

1. MFID

2. Name of legal reviewer

3. Is there a difference of opinion between the legal representative, doctor or legal reviewer about whether we can or cannot respond to instructions? If yes, please explain.

4. Was it necessary to feed back to the legal representative where we could only respond to some of the instructions, or could not complete the report? If yes, please explain.

5. Are there points in the legal case which remain unaddressed due to limitations 
of remote evidence gathering or clinical concerns relating to the assessment being done remotely? If yes, please briefly explain the point/s and the limitations.

6. Are there any learning points to feed back to doctors, or any other issues to note? If yes, please briefly explain. 